\title{
Representative Image Selection for Data Efficient Word Spotting*
}

\author{
Florian Westphal ${ }^{1[0000-0002-2161-7371]}$, Håkan Grahn ${ }^{10000-0001-9947-1088]}$, and \\ Niklas Lavesson ${ }^{1,2[0000-0002-0535-1761]}$ \\ 1 Blekinge Institute of Technology, Karlskrona, Sweden \\ \{florian.westphal, hakan.grahn\}@bth.se \\ 2 Jönköping University, Jönköping, Sweden niklas.lavesson@ju.se
}

\begin{abstract}
This paper compares three different word image representations as base for label free sample selection for word spotting in historical handwritten documents. These representations are a temporal pyramid representation based on pixel counts, a graph based representation, and a pyramidal histogram of characters (PHOC) representation predicted by a PHOCNet trained on synthetic data. We show that the PHOC representation can help to reduce the amount of required training samples by up to $69 \%$ depending on the dataset, if it is learned iteratively in an active learning like fashion. While this works for larger datasets containing about 1700 images, for smaller datasets with 100 images, we find that the temporal pyramid and the graph representation perform better.
\end{abstract}

Keywords: word spotting · sample selection · graph representation · PHOCNet $\cdot$ active learning.

\section{Introduction}

Being able to search a collection of historical handwritten document images for occurrences of a particular word, for example a name, can be a great help to historians and genealogists in their work. Furthermore, it can create a more simplified access to historical documents for a broader public. This task of searching for words in images either by string or by example is called word spotting [7]. In recent years, the performance of word spotting systems has greatly improved through the use of learning based approaches using convolutional neural networks (CNNs), for example in the work by Sudholt and Fink [25] or by Krishnan et al. [14]. However, one drawback of these approaches is the large amount of labeled data required to train these approaches.

In this paper, we explore sample selection as one possible way to improve the word spotting performance of a learning based system without increasing the number of labeled samples. This will make it possible to reduce the amount of data to label without compromising performance. The main idea is to select

\footnotetext{
* This work is part of the research project "Scalable resource-efficient systems for big
} data analytics" funded by the Knowledge Foundation (grant: 20140032) in Sweden. 
word images from an unlabeled set of images, which represent different aspects of the target collection as well as possible, given the limitation in the number of samples to select. This diversity in selected samples should lead to a better word spotting performance, given the same number of images, compared to randomly selecting images to label. In order to achieve diversity in the aspects relevant for word spotting, it is important to represent the unlabeled word images in a suitable way to facilitate the selection of such a diverse training set.

The main contribution of this paper is the proposal and evaluation of three different word image representations for sample selection. The evaluated representations are a temporal pyramid representation based on foreground pixel counts, a graph representation based on word graphs and a Pyramidal Histogram of Characters (PHOC) representation based on predicted PHOCs.

We show that it is possible, depending on the dataset, to reduce the required amount of training data by up to $69 \%$ without leading to a statically significant reduction in word spotting performance by learning to extract a suitable PHOC representation of word images using an active learning [20] approach. While this approach works well when around 1700 word images can be labeled, we show that the temporal pyramid and the graph representation can perform as good or better than random sampling for small dataset sizes of 100 images.

\section{Related Work}

While sample selection has not been applied to word spotting, it has been used for other document analysis tasks, such as character recognition and binarization. For character recognition, Rayar et al. [19] show that by selecting training samples based on the bridge vectors in a relative neighborhood graph it is possible to reduce the size of the original training set by almost $63 \%$ without reducing the recognition accuracy. However, one drawback of this approach is that it relies on label information in the original training set. Krantz and Westphal [13] follow a similar strategy for selecting training samples for image binarization using a relative neighborhood graph. In contrast to Rayar et al., their approach avoids the need for a labeled set to choose from by creating pseudo labels through clustering. In this way, they reduce the training set on average by $49.5 \%$, which leads to a decrease in binarization performance by $0.55 \%$.

Other methods, which have been used to increase the performance of word spotting approaches given only a limited number of training samples are data augmentation and pre-training on synthetic data. One possible augmentation strategy for word spotting is to apply different image transformations, such as shear, rotation and translation to the image, as has been proposed by Sudholt and Fink [25]. Gurjar et al. [11] have shown that pre-training a CNN based word spotting approach with the synthetic dataset by Krishnan and Jawahar [15] can achieve a reasonable word spotting performance, even with only few training samples. Since the achieved improvements for both of these methods are independent from the particular training set, it is likely that improvements achieved through the application of augmentation, pre-training and sample selection will 
add up. Thus, the sample selection approaches evaluated in this paper will add an additional way to reduce the amount of training data required for training a word spotting model on top of augmentation and pre-training.

\section{Background}

\subsection{Word Spotting with PHOCNet}

One CNN based approach to word spotting, which allows query by example, as well as query by string scenarios, is PHOCNet [24]. This neural network based on the VGG16 architecture [21] predicts the pyramidal histogram of characters (PHOC) [2] from a given input image. The PHOC is a vector of binary values, which indicate the presence or absence of characters from a predefined character set in different parts of a word. Since the PHOC can be derived from a string, it is possible to search for specific word images by predicting their PHOCs and then identifying those word images whose PHOCs are most similar to the generated search PHOC. Similarly, in a query by example scenario, the PHOC of the query image can be predicted and can then be used to find word images with similar PHOCs.

\subsection{Graph Matching}

Graph matching is a commonly used approach in pattern recognition $[6,10]$ and has been applied also to word spotting [23]. The main idea here is to represent a word image as a graph, as shown in Fig. 1. By computing the graph edit distance between this search graph and other word graphs, it is possible to search a dataset of word images for the most similar instances.

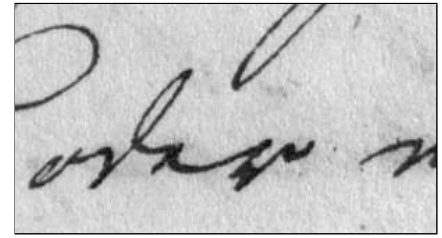

(a) Word Image

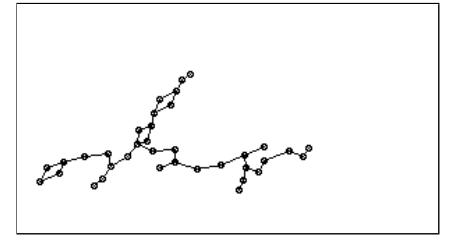

(b) Extracted Graph

Fig. 1. Word graph for one image from the Alvermann Konzilsprotokolle dataset [18].

For extracting graphs from word images, we use the projection based approach by Stauffer et al. [22]. This approach identifies first the nodes of a graph by splitting the binarized word image into segments using horizontal and vertical projection profiles, identifying gaps between characters. These segments are further subdivided horizontally and vertically in predefined intervals $D_{h}$ and 
$D_{v}$. The center of mass of each of these sub-segments is chosen as one node of the graph. Undirected edges are added between two nodes if their respective segments are connected by the lines of the skeletonized word.

Since computing the exact graph edit distance is an NP-hard problem, efficient graph matching requires suitable approximation algorithms. One of these algorithms is the Hausdorff edit distance (HED) [9]. The HED represents a lower bound to the exact graph edit distance and its computation has a quadratic time complexity. This is achieved by matching nodes and edges between the graphs independently, without considering other node or edge assignments.

\subsection{Sample Selection with Iterative Projection and Matching (IPM)}

While there are many different sampling strategies, such as K-medoids sampling, dissimilarity-based sparse subset selection [8], structured sparse dictionary selection [26] or representative selection on a hypersphere [27], in this paper, we focus on the iterative projection and matching (IPM) approach by Zaeemzadeh et al. [29]. In this approach, sample selection is achieved by reducing an $M \times N$ matrix $\mathbf{A}$ of $M$ training sample representations $\mathbf{a} \in \mathbb{R}^{N}$ to a $K \times N$ matrix containing $K$ selected samples. All vectors a representing the training samples are normalized, denoted as ã, to lie on the unit sphere. The resulting matrix of unit vectors is denoted as $\tilde{\mathbf{A}}$. The algorithm picks iteratively samples by first finding the most representative direction for the current matrix $\tilde{\mathbf{A}}$ in the unit sphere. Second, the unit vector $\tilde{\mathbf{a}}$ with the smallest angle to the chosen direction is selected and all unit vectors in $\tilde{\mathbf{A}}$ are projected onto the null space of the selected sample, effectively creating a lower dimensional subspace from which the following sample will be chosen. This process is repeated until $K$ samples were selected.

\section{Sample Selection for Word Spotting}

\subsection{Sample Selection Pipeline}

We select training samples from an unlabeled set of word images, as shown in Fig. 2, by first extracting a sample representation for each word image. Since this representation is the base for the selection process, it is important that it characterizes the word in the processed word image in such a way that it becomes distinguishable from images of other words. This makes it possible to select a set of images of different words, which is the dimension of diversity relevant for the learning task, i.e., PHOC prediction. In this paper, we evaluate three different word image representations, viz. a temporal pyramid representation (Section 4.2), a graph representation (Section 4.3), and a PHOC representation (Section 4.4).

After extracting the respective representations, we compute the pairwise distances between all word image representations using a suitable distance metric. In this way, a word image $x_{i}$ is represented as a vector $\mathbf{x}_{\mathbf{i}} \in \mathbb{R}_{+}^{N}$ containing 


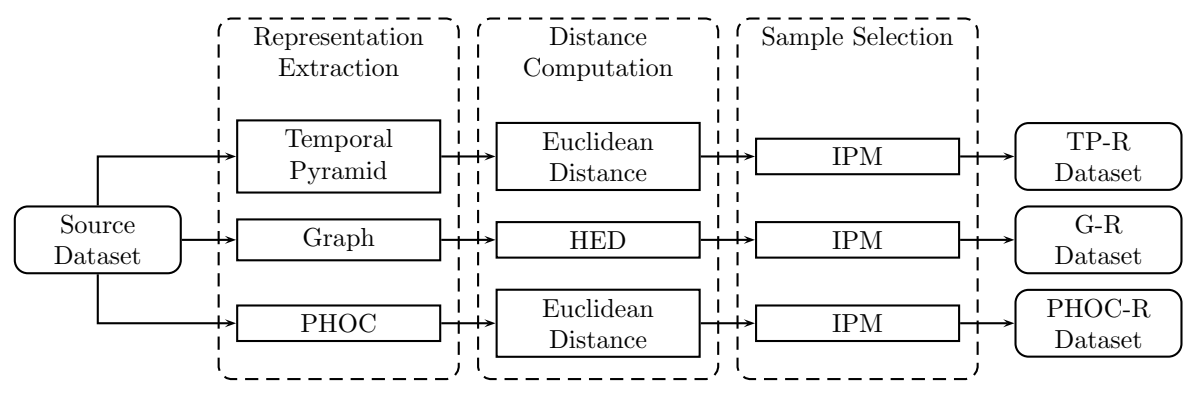

Fig. 2. Sample selection pipeline for the three evaluated word image representations.

its distance to all $N$ word images. The $N \times N$ distance matrix $\mathbf{D}$ is then used as input to IPM [29] to select the $K$ most representative samples. One advantage of representing the word images as vector of distances is that it leads to an $N$ dimensional vector space with $N>K$. This is beneficial for IPM, since the IPM algorithm effectively reduces the dimensionality of its input matrix through projection for every chosen sample. The IPM algorithm then yields the most representative $K$ samples given the provided distance matrix $\mathbf{D}$.

\subsection{Temporal Pyramid Representation (TP-R)}

One simplistic approach to represent a word image is the proposed temporal pyramid representation (TP-R). This approach represents a word image as vector of pixel counts in different segments of the word image.

In order to ensure that the pixel counts represent the actual word in the image, we remove noise by first binarizing the image. Furthermore, we remove possible word fragments from adjacent words in the image by finding at most four appropriate seams to cut away fragments close to the four borders of the image using the seam carving algorithm [3]. Lastly, we resize the word image to remove the created white space around the word, as illustrated in Fig. 3.

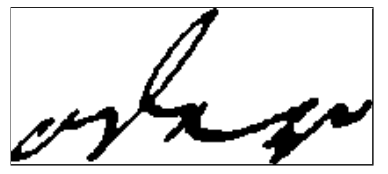

(a) Cleaned Image

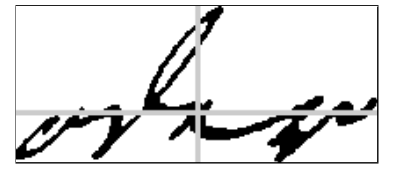

(b) Segments for $n=2$

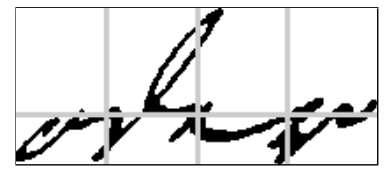

(c) Segments for $n=4$

Fig. 3. Word graph for one image from the Alvermann Konzilsprotokolle dataset [18].

Similar to temporal pyramid pooling [25], we split the input vertically into $n$ segments for different values of $n$. However, we also split the input image 
horizontally at the vertical center of mass to make it possible to distinguish ascenders and descenders. The resulting segments for $n=2$ and $n=4$ are shown in Fig. 3. The sums of pixels for each of the created segments are normalized by dividing them by the total number of foreground pixels in the word image and combined into a single vector representing the image. For the example given in Fig. 3, this results in a 12-dimensional vector. The distance between different word images can then easily be computed using the Euclidean distance. Here, we use the Euclidean distance as it appeared to have a slightly better performance in initial experiments than the Cosine similarity.

The main advantage of this representation is that it is fast to extract and that it is fast to compute the distance between two representations. One possible disadvantage is that this approach is susceptible to variations in writing style, such as slant, skew or spacing variations within a word.

\subsection{Graph Representation (G-R)}

Another possible word image representation is its graph representation (G-R). We extract the graph by first pre-processing the word image as described in Section 4.2 and then deriving the word graph from the cleaned image using the projection based approach by Stauffer et al. [22].

Since the graph representations cannot serve as input to the used sample selection algorithm, we compute the pairwise distances between the word graphs using the Hausdorff edit distance (HED) [9]. For the HED computation we use the cost model proposed by Stauffer et al. [23]. This model defines a node insertion and deletion cost $\tau_{v}$, an edge insertion and deletion cost $\tau_{e}$, as well as a parameter $\alpha$ to trade-off node edit costs with edge edit costs and a parameter $\beta$ to trade-off shifts in node location in horizontal direction with shifts in vertical direction when computing node substitution costs.

The main advantage of this representation is that it captures the shape of each character in the word and is thus more resistant to variations in writing style than TP-R. However, while it is fast to extract the graph representation from a word image, computing the HED requires considerably longer computation times than computing the Euclidean distance.

\subsection{PHOC Representation (PHOC-R)}

We extract the PHOC representation (PHOC-R) of a word image by predicting it using a PHOCNet pre-trained on the synthetic dataset by Krishnan and Jawahar [14]. While it is necessary to use a PHOCNet trained in such a way when no training samples have been selected and labeled, the PHOCNet can be tuned to the target dataset, as soon as a small set of word images has been selected. In this way, the PHOC representation can get more suitable for the target dataset the more samples have been labeled. Thus, this representation facilitates active learning [20]. After extracting the PHOCs, the pairwise Euclidean distances are computed, as shown in Fig. 2. Here, the Euclidean distance is used instead of the 
Cosine similarity, which is common in PHOC based retrieval, since initial tests have shown a better sample selection performance for the Euclidean distance.

The main advantage of this representation is that it is closely aligned with the learning task and that it can improve based on the number of training samples available. Furthermore, the distance computation between two representations is fast. One possible disadvantage of this approach is that it requires the PHOCNet to be retrained whenever more training data becomes available. Since it is reasonable to select only few samples initially, when the PHOCNet's performance can be expected to be poor, this can lead to several time intensive training runs until the desired training set size is reached.

\section{Experiment Design}

\section{$5.1 \quad$ Experiment Setup}

We conduct all experiments in this paper using the PHOCNet implementation ${ }^{3}$ by Sudholt and Fink [24]. For the sample selection, we use the IPM implementation $^{4}$ by Zaeemzadeh et al. [29] and we compute the HED between graphs using the HED implementation in GEDLIB ${ }^{5}$ by Blumenthal et al. [4,5], which we modified to support the cost model by Stauffer et al. [23]. The code used to conduct all experiments as well as the raw data is available online ${ }^{6}$. All experiments were conducted on a computer with an Intel i9-7900X CPU @ 3.3GHz, 32 GB DDR4 RAM and two Nvidia GeForce GTX 1080 Ti.

We evaluate the different word image representations using the Alvermann Konzilsprotokolle (AK) dataset and the Botany dataset from the ICFHR2016 Handwritten Keyword Spotting Competition (H-KWS) [18], as well as the George Washington (GW) dataset by Lavrenko et al. [16]. For the two H-KWS datasets, we use their respective Train II partition as source dataset to select samples from and we use the size of their respective Train I partition as the maximum number of samples to select. This means for the Botany dataset, for example, that the largest selected training dataset with 1684 images is chosen from a dataset with 3611 images. Since there is no official partitioning of the George Washington dataset into train, validation and test set, we use the partitioning for the first of the four folds used by Almazán et al. [2]. We combine the train and validation set into one source dataset and use the size of the train dataset as the maximum number of samples to select. For all datasets, we select training sets with 100, 500 , and the dataset specific maximum number of samples.

Since the extraction of TP-R and G-R requires binarized input images, we binarize all word images using Howe's binarization algorithm [12]. We predict the required binarization parameters using the approach by Westphal et al. [28], which has been trained on the dataset of the 2013 document image binarization

\footnotetext{
3 https://github.com/ssudholt/phocnet

${ }^{4}$ https://github.com/zaeemzadeh/IPM

${ }^{5}$ https://github.com/dbblumenthal/gedlib

${ }^{6}$ https://github.com/FlorianWestphal/DAS2020
} 
contest [17]. After visual inspection of one image, we adjust one of the predicted parameters for the Alvermann Konzilsprotokolle dataset and the George Washington dataset to increase the recall.

Apart from the pre-processing, the graph extraction and HED computation requires a number of dataset specific parameters, such as $D_{v}$ and $D_{h}$ for the graph extraction and $\tau_{v}, \tau_{e}, \alpha$, and $\beta$ for the HED computation. In our experiments, we use the parameters suggested by Stauffer et al. [23] for each of the respective datasets. Since these parameters were obtained through parameter tuning on labeled samples of the respective datasets, the obtained results have to be seen as an upper bound for the performance. This is the case, since we assume that no labeled data is available before performing the sample selection.

In order to make use of the ability of PHOC-R to adapt to a given dataset by training the used PHOCNet, we initially select only 50 images using the PHOCs predicted by a PHOCNet pre-trained on the HW-SYNTH dataset by Krishnan and Jawahar [15]. Then this PHOCNet is trained further using the selected 50 images. Based on the PHOCs predicted by this network, another 50 images are selected to obtain the first training set containing 100 images. This is continued by training the first PHOCNet, which was trained on the HW-SYNTH dataset, on the selected 100 images. Once the network is trained, its predicted PHOCs are used to select 400 additional images to obtain the next largest training set containing 500 images. In a similar fashion, the samples for the dataset specific maximum number of samples are chosen.

Apart from using the three evaluated word image representations for sample selection, we also use random sample selection as baseline for comparison. In order to obtain a more reliable result, we randomly select three training sets for each training set size and each of the used datasets. For this selection approach, we report the average results over these three training sets. Thus, we evaluate in total four sample selection approaches, viz. TP-R, G-R, PHOC-R and Random.

\subsection{Training Setup}

In this paper, we use a PHOCNet with temporal pyramid pooling (TPP) layer, as described by Sudholt and Fink [25]. As described by Gurjar et al. [11], we train the PHOCNet using stochastic gradient descend and use binary cross entropy as loss function when training to predict PHOCs.

For the selected training sets containing 100 and 500 images, we pre-train the network using the HW-SYNTH dataset [15] for 80000 weight update steps with a batch size of 10 , a momentum of 0.9 , a weight decay of $5 \cdot 10^{-5}$ and an initial learning rate of $10^{-4}$, which is decreased after 70000 steps to $10^{-5}$. The training is continued with the same momentum vector using the selected training samples at a constant learning rate of $10^{-5}$. For the training sets containing 100 images, this training is continued for 40000 steps, while for those sets containing 500 images training is continued for 70000 steps. These training durations have been chosen to ensure convergence on all datasets. When using the dataset specific maximum number of samples, no pre-training is used and the PHOCNet 
is trained from scratch for 80000 weight update steps with the same parameters as when training on the HW-SYNTH dataset.

We extract the character set, which is used to define the dataset specific PHOC vector, for each dataset from all transcriptions in its source dataset. Here, we make use of all available transcriptions, since it is reasonable to assume that a suitable character set can be derived given knowledge about the dataset for which the PHOCNet should be trained. In this paper, we perform case insensitive word spotting. Therefore, we convert all ground truth labels to upper case. Since the PHOC descriptors are dataset specific, the HW-SYNTH pre-training has to be performed for each dataset.

In order to increase the generalization performance of the trained PHOCNet, we use data augmentation. While we augment the images of the HW-SYNTH dataset by varying their size, as described by Gurjar et al. [11], we use the augmentation strategy proposed by Sudholt and Fink [25] for the evaluated datasets. Depending on the number of weight update steps, we generate 400000 or 500000 augmented samples for each training set. When the original training set size is only 100 images, we generate only 400000 images, since this is the maximum number of images the network can process in 40000 update steps.

\subsection{Evaluation Procedure}

We evaluate the word spotting performance of the trained PHOCNets using the official evaluation dataset in case of the Alvermann Konzilsprotokolle dataset and the Botany dataset. For the George Washington dataset, we follow the evaluation procedure by Almazán et al. [2]. This means that we use the transcriptions of all word images in the test dataset as query strings for the query by string (QbS) case. For the query by example (QbE) case, we use all test images as query image, as long as at least one other image of the same word is present in the test dataset. In both cases, the images are retrieved from the test. In the QbE case, the respective query image is removed from the retrieval result.

We evaluate the retrieval performance using the mean average precision (mAP), which is the average over the average precisions (APs) for all evaluation queries. The average precision $A P_{q}$ for a query $q$ is defined as follows:

$$
A P_{q}=\frac{\sum_{i=1}^{n} P\left(R_{q, i}, E_{q}\right) \cdot I\left[r_{i} \in E_{q}\right]}{\left|E_{q}\right|}
$$

Here, $R_{q, i} \subseteq R_{q, n}$ denotes the set of the first $i$, out of $n$, retrieved elements for the query $q$. The set of relevant or expected elements for $q$ is denoted by $E_{q}$ and $I[\cdot]$ is the indicator function, which is 1 if the $i$ th retrieved element $r_{i} \in R_{q, i}$ is relevant with respect to $q$ and 0 otherwise. The function $P(R, E)$ returns the precision given the set of retrieved elements $R$ and the set of relevant or expected elements $E$. It is defined as follows:

$$
P(R, E)=\frac{|R \cap E|}{|R|}
$$




\section{Results and Analysis}

Tables 1, 2, and 3 show the word spotting results in $\mathrm{mAP}$ for the QbE and QbS case for all three training set sizes for the four evaluated sample selection approaches TP-R, G-R, PHOC-R and Random. Furthermore, the last two rows of those tables show the $\mathrm{QbE}$ and $\mathrm{QbS}$ results for the case that the PHOCNet is trained on all images in the source dataset. Fig. 4 shows the relative number of unique words within the selected training sets with respect to the total number of unique words in the respective source set in relation to the relative mAP results. These results are relative to the $\mathrm{mAP}$ achieved by training on the whole source dataset. In this way, it is possible to show the mAP results for all datasets, selection approaches, query types and training set sizes in one scatter plot.

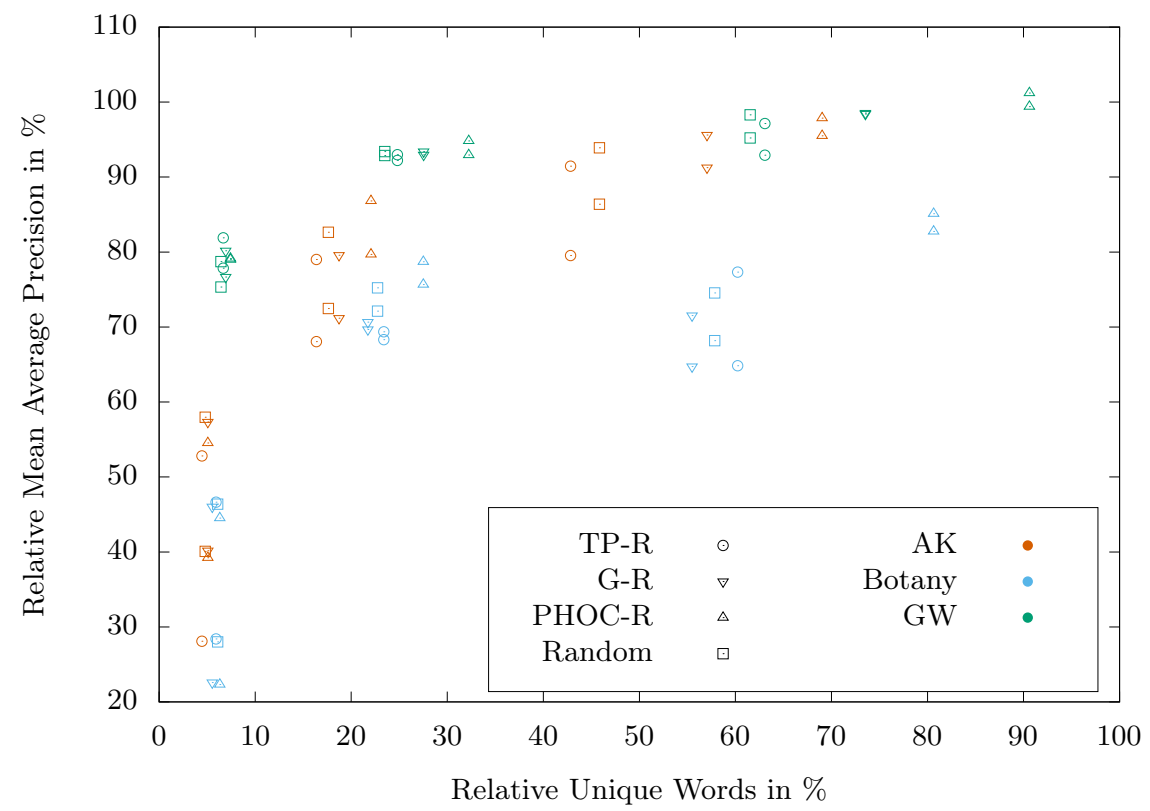

Fig. 4. Relationship between the relative number of unique selected words and the achieved relative mean average precision for the four evaluated sample selection approaches and the three evaluated datasets.

In order to compare the results for the four selection approaches for each training set size and each of the two query types, i.e., $\mathrm{QbE}$ and $\mathrm{QbS}$, we perform a one-way repeated measures analysis of variances (ANOVA) on the average precisions (APs) obtained from each evaluation query. We use this statistic test, since the same query, i.e., the subject or independent variable, is answered by PHOCNets trained using differently selected training sets, i.e., the treatment, 
resulting in AP results, i.e., the dependent variable. This setup corresponds to the one-way repeated measures design. We avoid making assumptions about the distribution of the obtained AP results by applying a rank transform to the APs of the evaluated approaches before applying the ANOVA test.

If the applied ANOVA test indicates a statistically significant difference between the APs obtained for the four sample selection approaches, we perform a pairwise Wilcoxon rank sum test with Holm correction to identify between which approaches exist statistically significant differences. The application of the Holm correction to the pairwise tests is important to avoid the multiple comparisons problem. We show the results of these pairwise comparisons in the tables in form of score values indicated in brackets behind each of the mAP results. These score values are computed by adding one to the score of a method if it is statistically significantly better than another method at the $p<0.05$ level and we reduce the score by one if a method is statistically significantly worse than another method. Therefore, the score values within one comparison group add up to zero. If the ANOVA test does not indicate any statistically significant differences between approaches at the $p<0.05$ level, the score value is left blank.

Table 1. Word spotting results for the Alvermann Konzilsprotokolle dataset in $\mathrm{mAP}(\%)$ and the corresponding score values indicating statistically significantly better $(+1)$ or worse $(-1)$ results at the $p<0.05$ level.

\begin{tabular}{r|ccccc}
\hline & TP-R & G-R & PHOC-R & Random & All \\
\hline $100 \mathrm{QbE}$ & $51.08(-1)$ & $55.45( \pm 0)$ & $52.73( \pm 0)$ & $56.07(+1)$ & - \\
$\mathrm{QbS}$ & $26.88(-2)$ & $38.45(+1)$ & $37.55( \pm 0)$ & $38.36(+1)$ & - \\
\hline $500 \mathrm{QbE}$ & $76.41(-1)$ & $76.95(-1)$ & $83.96(+3)$ & $79.91(-1)$ & - \\
$\mathrm{QbS}$ & $65.13(-1)$ & $68.13( \pm 0)$ & $76.27(+1)$ & $69.36( \pm 0)$ & - \\
\hline $1849 \mathrm{QbE}$ & $88.45(-2)$ & $92.47(+2)$ & $94.64(+2)$ & $90.80(-2)$ & - \\
$\mathrm{QbS}$ & $76.12(-1)$ & $87.35(+1)$ & $91.41(+2)$ & $82.67(-2)$ & - \\
\hline $5968 \mathrm{QbE}$ & - & - & - & - & 96.71 \\
$\mathrm{QbS}$ & - & - & - & - & 95.71 \\
\hline
\end{tabular}

Based on the results for the Alvermann Konzilsprotokolle (AK) dataset in Tab. 1, one can see that TP-R performs statistically significantly worse than at least one other method for all query types and training set sizes. One possible reason for this could be variations in writing style, such as varying spacing between characters or differences in size for certain characters in a word, which lead to different TP-Rs for the same word, making it more likely that images of the same word are selected. Fig. 4 confirms this by showing that for the AK dataset TP-R selects a lower ratio of unique words per selected training set than any other selection approach.

For G-R and PHOC-R, one can see from Tab. 1 that these approaches perform better compared to the other two approaches, the more training samples 
are selected. While this is to be expected for PHOC-R, since its word image representation improves with the number of available training samples, it is surprising for G-R. One explanation for this result may be that it is not G-R, which improves, but rather that random selection fails to make use of the possibility to select a more diverse sample when the training set is large. This can be seen in Fig. 4, which shows that G-R selects nearly the same ratio of unique words as TP-R and Random for the two smaller training sets, but selects a higher ratio of unique words than both of them for the largest training set.

Lastly, we test for statistically significant differences between the word spotting results obtained by training on the largest training set selected using G$\mathrm{R}$ and PHOC-R and the results obtained using the whole source dataset for training. We find that there is no statistically significant difference between the training set selected using PHOC-R and the source dataset. This indicates that by using PHOC-R, it is possible to reduce the training set for the AK dataset by $69 \%$ without statistically significant reduction in word spotting performance.

Table 2. Word spotting results for the Botany dataset in mAP (\%) and the corresponding score values indicating statistically significantly better $(+1)$ or worse $(-1)$ results at the $p<0.05$ level.

\begin{tabular}{r|ccccc}
\hline & TP-R & G-R & PHOC-R & Random & All \\
\hline $100 \mathrm{QbE}$ & $36.73(-)$ & $36.24(-)$ & $35.06(-)$ & $36.52(-)$ & - \\
$\mathrm{QbS}$ & $23.83( \pm 0)$ & $18.94(-1)$ & $18.73(-1)$ & $23.50(+2)$ & - \\
\hline $500 \mathrm{QbE}$ & $54.62( \pm 0)$ & $54.86( \pm 0)$ & $59.59( \pm 0)$ & $56.80( \pm 0)$ & - \\
$\mathrm{QbS}$ & $57.31( \pm 0)$ & $59.27( \pm 0)$ & $66.04( \pm 0)$ & $63.12( \pm 0)$ & - \\
\hline $1684 \mathrm{QbE}$ & $60.89( \pm 0)$ & $56.33(-1)$ & $65.16(+2)$ & $58.70(-1)$ & - \\
$\mathrm{QbS}$ & $54.40(-1)$ & $54.31(-1)$ & $71.41(+3)$ & $57.21(-1)$ & - \\
\hline $3611 \mathrm{QbE}$ & - & - & - & - & 78.75 \\
$\mathrm{QbS}$ & - & - & - & - & 83.90 \\
\hline
\end{tabular}

Similar to the AK dataset, one can see for the results of the Botany dataset in Tab. 2 that the word spotting performance of PHOC-R improves compared to the other three approaches, the more training samples are available. However, we do not see a similar improvement for G-R. One reason for this may be the larger variation in writing styles in the Botany dataset leading to less unique words to be selected, as shown in Fig. 4. However, this cannot be the only explanation, since such variations should affect TP-R even more than G-R. Another possible aspect could be binarization errors, which affect G-R more than TP-R, since they can make the graph representations of the same word appear to be different. TP-R is presumably more robust towards binarization issues, due to the normalization of the word image's pixel counts.

When comparing the word spotting performance of a PHOCNet trained on the complete source dataset with the performance of a model trained on the 
largest training set selected using PHOC-R, we find the former to perform statistically significant better than the latter. This may be due to the large writing style variations in the Botany dataset, which lead to the necessity for larger training datasets.

Table 3. Word spotting results for the George Washington dataset in mAP (\%) and the corresponding score values indicating statistically significantly better $(+1)$ or worse $(-1)$ results at the $p<0.05$ level.

\begin{tabular}{r|ccccc}
\hline & TP-R & G-R & PHOC-R & Random & All \\
\hline $100 \mathrm{QbE}$ & $77.05(+3)$ & $72.13(-2)$ & $74.47(+1)$ & $74.07(-2)$ & - \\
$\mathrm{QbS}$ & $71.68(+1)$ & $73.81(+1)$ & $72.73(+1)$ & $69.38(-3)$ & - \\
\hline $500 \mathrm{QbE}$ & $86.77( \pm 0)$ & $87.88(+1)$ & $87.45(+1)$ & $87.39(-2)$ & - \\
$\mathrm{QbS}$ & $85.62( \pm 0)$ & $85.60( \pm 0)$ & $87.32(+1)$ & $86.00(-1)$ & - \\
\hline $1823 \mathrm{QbE}$ & $91.39( \pm 0)$ & $92.72( \pm 0)$ & $95.22(+3)$ & $92.50(-3)$ & - \\
$\mathrm{QbS}$ & $85.55(-2)$ & $90.62(+2)$ & $91.53(+2)$ & $87.67(-2)$ & - \\
\hline $3645 \mathrm{QbE}$ & - & - & - & - & 94.09 \\
$\mathrm{QbS}$ & - & - & - & - & 92.08 \\
\hline
\end{tabular}

For the George Washington (GW) dataset, the relative word spotting performance for G-R and PHOC-R is comparable to the AK dataset with respect to the fact that their performance improves compared to the other two approaches, the more training samples are selected (cf. Tab. 3). However, TP-R performs better on this dataset than on the AK dataset, in particular when only 100 training samples are selected. This may be the case, since there is less variation in writing style in the GW dataset, which makes it easier for TP-R to select word images with more unique words. We compare the word spotting performance achieved by training on the source dataset with the performance achieved by training on the largest selected training sets. We find that there is no statistically significant difference between the performance of G-R, PHOC-R and the source dataset in the QbS case and that there is no statistically significant different between the performance of PHOC-R and the source dataset in the QbE case. Therefore, we conclude that using PHOC-R can help to reduce the amount of training data required for the GW dataset by almost $50 \%$.

\section{Conclusion}

In this paper, we have evaluated three different word image representations for unlabeled sample selection for word spotting. The evaluated representations are a temporal pyramid representation (TP-R), a graph based representation (G-R) and a pyramidal histogram of characters representation (PHOC-R). We have shown that, depending on the dataset, the use of PHOC-R can lead to a reduction in the number of required training samples of up to $69 \%$ without reducing 
the word spotting performance in a statistically significant way. We have argued that the main reason for PHOC-R's performance is that it learns to adjust its word image representation in an active learning like fashion. Thus, it performs best when a larger amount of training samples can be retrieved iteratively. For selecting smaller training sets, we have shown that G-R and TP-R can perform as well or better than random selection. We have pointed out that current limitations of these two word image representations may be due to binarization issues, as well as their susceptibility to variations in writing style. Possible future work would be to improve the used binarization method and to increase TP-R's robustness by applying skew and slant correction to the word images. Additionally, it may be interesting to consider and evaluate the use of different distance metrics for TP-R and PHOC-R. Since both representations have many dimensions, the Manhattan distance or fractional distance metrics may make the represented word images more distinguishable, as pointed out by Aggarwal et al. [1], which could result in a more suitable sample selection.

\section{References}

1. Aggarwal, C.C., Hinneburg, A., Keim, D.A.: On the surprising behavior of distance metrics in high dimensional space. In: International conference on database theory. pp. 420-434. Springer (2001)

2. Almazán, J., Gordo, A., Fornés, A., Valveny, E.: Word spotting and recognition with embedded attributes. IEEE Transactions on Pattern Analysis and Machine Intelligence 36(12), 2552-2566 (2014)

3. Avidan, S., Shamir, A.: Seam carving for content-aware image resizing. ACM Transactions on graphics (TOG) 26(3), 10-1-10-9 (2007)

4. Blumenthal, D.B., Boria, N., Gamper, J., Bougleux, S., Brun, L.: Comparing heuristics for graph edit distance computation. The VLDB Journal pp. 1-40 (2019)

5. Blumenthal, D.B., Bougleux, S., Gamper, J., Brun, L.: Gedlib: A c ++ library for graph edit distance computation. In: International Workshop on Graph-Based Representations in Pattern Recognition. pp. 14-24. Springer (2019)

6. Conte, D., Foggia, P., Sansone, C., Vento, M.: Thirty years of graph matching in pattern recognition. International Journal of Pattern Recognition and Artificial Intelligence 18(03), 265-298 (2004)

7. Doermann, D.: The indexing and retrieval of document images: A survey. Computer Vision Image Understanding 70(3), 287-298 (1998)

8. Elhamifar, E., Sapiro, G., Sastry, S.S.: Dissimilarity-based sparse subset selection. IEEE transactions on pattern analysis and machine intelligence 38(11), 2182-2197 (2015)

9. Fischer, A., Suen, C.Y., Frinken, V., Riesen, K., Bunke, H.: Approximation of graph edit distance based on hausdorff matching. Pattern Recognition 48(2), 331343 (2015)

10. Foggia, P., Percannella, G., Vento, M.: Graph matching and learning in pattern recognition in the last 10 years. International Journal of Pattern Recognition and Artificial Intelligence 28(01) (2014)

11. Gurjar, N., Sudholt, S., Fink, G.A.: Learning deep representations for word spotting under weak supervision. In: 13th IAPR International Workshop on Document Analysis Systems. pp. 7-12. IEEE (2018) 
12. Howe, N.R.: A laplacian energy for document binarization. In: 2011 International Conference on Document Analysis and Recognition. pp. 6-10. IEEE (2011)

13. Krantz, A., Westphal, F.: Cluster-based sample selection for document image binarization. In: International Conference on Document Analysis and Recognition Workshops. vol. 5, pp. 47-52. IEEE (2019)

14. Krishnan, P., Dutta, K., Jawahar, C.: Word spotting and recognition using deep embedding. In: 13th IAPR International Workshop on Document Analysis Systems. pp. 1-6. IEEE (2018)

15. Krishnan, P., Jawahar, C.V.: Matching handwritten document images. In: European Conference on Computer Vision. pp. 766-782. Springer (2016)

16. Lavrenko, V., Rath, T.M., Manmatha, R.: Holistic word recognition for handwritten historical documents. In: First International Workshop on Document Image Analysis for Libraries, 2004. Proceedings. pp. 278-287. IEEE (2004)

17. Pratikakis, I., Gatos, B., Ntirogiannis, K.: Icdar 2013 document image binarization contest (dibco 2013). In: 2013 12th International Conference on Document Analysis and Recognition. pp. 1471-1476. IEEE (2013)

18. Pratikakis, I., Zagoris, K., Gatos, B., Puigcerver, J., Toselli, A.H., Vidal, E.: Icfhr2016 handwritten keyword spotting competition (h-kws 2016). In: 15th International Conference on Frontiers in Handwriting Recognition (ICFHR). pp. 613-618. IEEE (2016)

19. Rayar, F., Goto, M., Uchida, S.: Cnn training with graph-based sample preselection: application to handwritten character recognition. In: 13th IAPR International Workshop on Document Analysis Systems. pp. 19-24. IEEE (2018)

20. Settles, B.: Active learning. Synthesis Lectures on Artificial Intelligence and Machine Learning 6(1), 1-114 (2012)

21. Simonyan, K., Zisserman, A.: Very deep convolutional networks for large-scale image recognition. In: Bengio, Y., LeCun, Y. (eds.) 3rd International Conference on Learning Representations (2015), http://arxiv.org/abs/1409.1556

22. Stauffer, M., Fischer, A., Riesen, K.: A novel graph database for handwritten word images. In: Joint IAPR Int. Workshops Statistical Techn. Pattern Recognition (SPR) Structural and Syntactic Pattern Recognition (SSPR). pp. 553-563 (2016)

23. Stauffer, M., Fischer, A., Riesen, K.: Keyword spotting in historical handwritten documents based on graph matching. Pattern Recognition 81, 240-253 (2018)

24. Sudholt, S., Fink, G.A.: Evaluating word string embeddings and loss functions for cnn-based word spotting. In: 14th IAPR International Conference on Document Analysis and Recognition. vol. 1, pp. 493-498. IEEE (2017)

25. Sudholt, S., Fink, G.A.: Attribute cnns for word spotting in handwritten documents. International Journal on Document Analysis and Recognition 21(3), 199218 (2018)

26. Wang, H., Kawahara, Y., Weng, C., Yuan, J.: Representative selection with structured sparsity. Pattern Recognition 63, 268-278 (2017)

27. Wang, H., Yuan, J.: Representative selection on a hypersphere. IEEE Signal Processing Letters 25(11), 1660-1664 (2018)

28. Westphal, F., Grahn, H., Lavesson, N.: Efficient document image binarization using heterogeneous computing and parameter tuning. International Journal on Document Analysis and Recognition (IJDAR) 21(1-2), 41-58 (2018)

29. Zaeemzadeh, A., Joneidi, M., Rahnavard, N., Shah, M.: Iterative projection and matching: Finding structure-preserving representatives and its application to computer vision. In: Proceedings of the IEEE Conference on Computer Vision and Pattern Recognition. pp. 5414-5423 (2019) 